\title{
Resonant transmission of light through surface plasmon structures
}

Kemal Gurel, Burkan Kaplan, Hasan Guner, Mehmet Bayindir, and Aykutlu Dana

Citation: Appl. Phys. Lett. 94, 233102 (2009);

View online: https://doi.org/10.1063/1.3151828

View Table of Contents: http://aip.scitation.org/toc/apl/94/23

Published by the American Institute of Physics

\section{Articles you may be interested in}

Surface plasmon resonance and surface-enhanced Raman scattering sensing enabled by digital versatile discs

Applied Physics Letters 100, 041116 (2012); 10.1063/1.3679682

High performance optical absorber based on a plasmonic metamaterial

Applied Physics Letters 96, 251104 (2010); 10.1063/1.3442904

Nanoimprint lithography

Journal of Vacuum Science \& Technology B: Microelectronics and Nanometer Structures Processing, Measurement, and Phenomena 14, 4129 (1998); 10.1116/1.588605

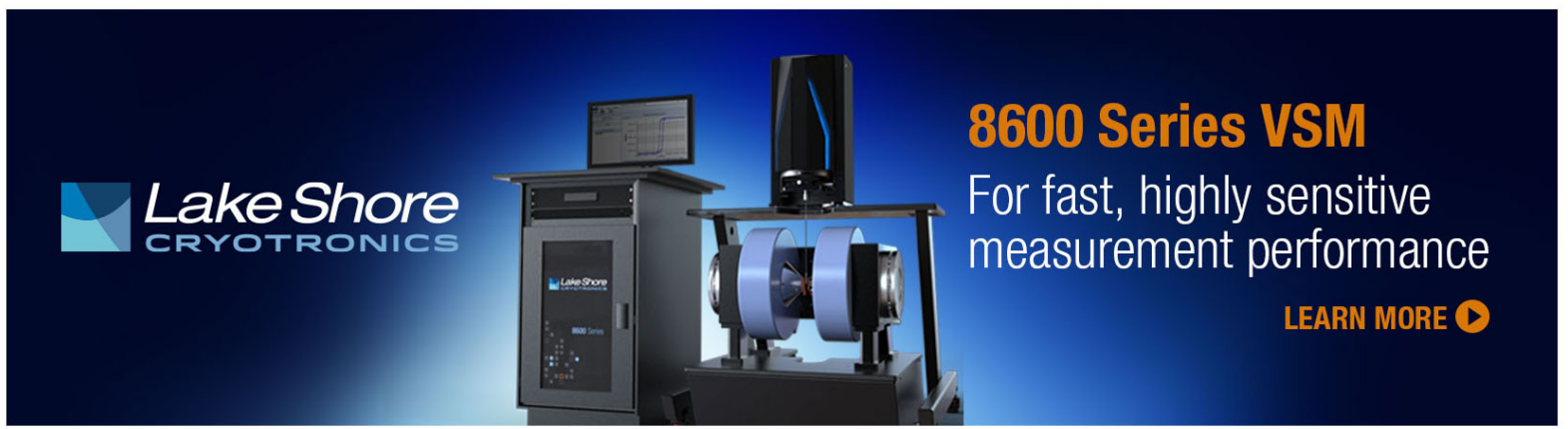




\title{
Resonant transmission of light through surface plasmon structures
}

\author{
Kemal Gurel, ${ }^{1}$ Burkan Kaplan, ${ }^{1}$ Hasan Guner, ${ }^{1}$ Mehmet Bayindir, ${ }^{1,2, a)}$ and Aykutlu Dana ${ }^{1, b)}$ \\ ${ }^{1}$ UNAM-Institute of Materials Science and Nanotechnology, 06800 Ankara, Turkey \\ ${ }^{2}$ Department of Physics, Bilkent University, 06800 Ankara, Turkey
}

(Received 14 March 2009; accepted 16 May 2009; published online 8 June 2009)

\begin{abstract}
Plasmonics enables the realization of new optical components. Here, we report yet another plasmonic component based on a pair of surfaces displaying grating coupled plasmon enhanced transmission. We observe high quality factor transmission peaks as high as 100 through our plasmonic filter based on gratings obtained directly from optical storage disks. Wavelength and polarization dependent transmission is also demonstrated in the visible and infrared portions of the spectrum. The resonance wavelength of this filter can be tuned by simply changing the angle of incidence. Numerical calculations agree well with measurements. Our work can open up directions toward disposable optical components such as filters and polarizers. (c) 2009 American Institute of Physics. [DOI: 10.1063/1.3151828]
\end{abstract}

In recent years, plasmonics has attracted a great deal of attention due to its important potential technological applications in sensing, imaging and information processing. ${ }^{1,2} \mathrm{Al}-$ though plasmonic structures have been extensively studied for better understanding of fundamental physical phenomenon, optoelectronic device applications generally focused on sensors. ${ }^{3}$ Since the pioneering works of Ebbesen et al. ${ }^{4}$ and Ghaemi et al. ${ }^{5}$ transmission of electromagnetic waves through thin metal films via plasmons has widely been studied. Plasmon resonance enhanced transmission through subwavelength holes, hole arrays or periodically corrugated metal surfaces have been investigated both theoretically and experimentally. ${ }^{6-10}$ The resonant transmission peaks due to interacting plasmonic structures (weak coupling regime) have been observed in silver films containing an array of Silicon spheres. ${ }^{11}$ Optical properties of the periodic arrays of defects (voids) in mesoporous metals ${ }^{12}$ and two-dimensional metallic quasiphotonic crystals ${ }^{13}$ have also been investigated.

In this article, we report observation of sharp transmission resonances in coupled surface-plasmon on metallized grating structures which are obtained from optical storage disks. A filter based on plasmon enhanced transmission (PET) in metal coated coupled-grating structures is proposed and experimentally demonstrated. The filter consists of two metallized gratings placed back to back, as shown in Fig. 1(a). In an optimized layer structure with sufficiently thin metal layer, light is partially transmitted through the metal layer and is partially diffracted or re-emitted into different orders with different angles of propagation. For our proposed structure, the transmitted orders travel inside the substrate and are incident onto an identical grating on the other face of the substrate. Due to the symmetry of the device, components of the light wave that are generated through plasmonic emission recouple to the plasmon mode and a second event of PET takes place at the second grating. The distance between the two surfaces carrying the gratings, $t$, is very large compared to the plasmon propagation length and free space wavelength $(t \gg \xi, \lambda)$. Under this condition, the reflected waves from the second surface do not interfere with (regen-

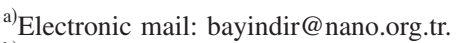

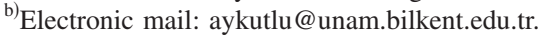

erate) the plasmons on the first surface, hence our analysis remains valid.

Figure 1(b) shows the calculated field distribution through the device for wavelength $\lambda=580 \mathrm{~nm}$ and angle of incidence $\theta_{i}=15^{\circ}(\Lambda=740 \mathrm{~nm}$ and $\Lambda \ll t)$. It can be seen that the electromagnetic (EM) waves are re-emitted from second grating layer at the same angle of incidence as the incoming wave. Even though the dielectric layer thickness between the gratings is on the order of $1 \mathrm{~mm}$ in real device structures, it is assumed to be $10 \mu \mathrm{m}$ to avoid computational difficulties in the finite difference time domain (FDTD) simulations. In this case, $t$ is much larger than the wavelength of light of incident beam, but it is comparable to the plasmon propagation length.
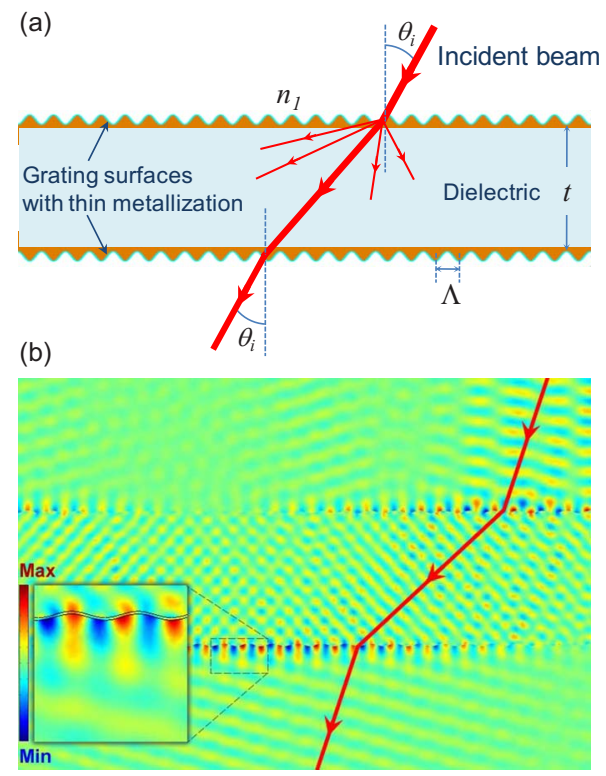

FIG. 1. (Color online) (a) Surface plasmons on coupled nanostructured gratings. The plasmonic filter consists of two metallized grating surfaces placed in a symmetrical fashion with a dielectric layer in between. Due to PET, light is radiated within the substrate into different diffraction orders. At the back side, as the same matching condition holds, light again couples to the plasmon mode and is radiated to vacuum at the same angle as the angle of incidence to the first surface. (b) Calculated $H_{z}$ component of EM wave through coupled plasmonic structure agrees well with proposed scheme. Inset shows the plasmonic radiation from sinusoidal grating. 

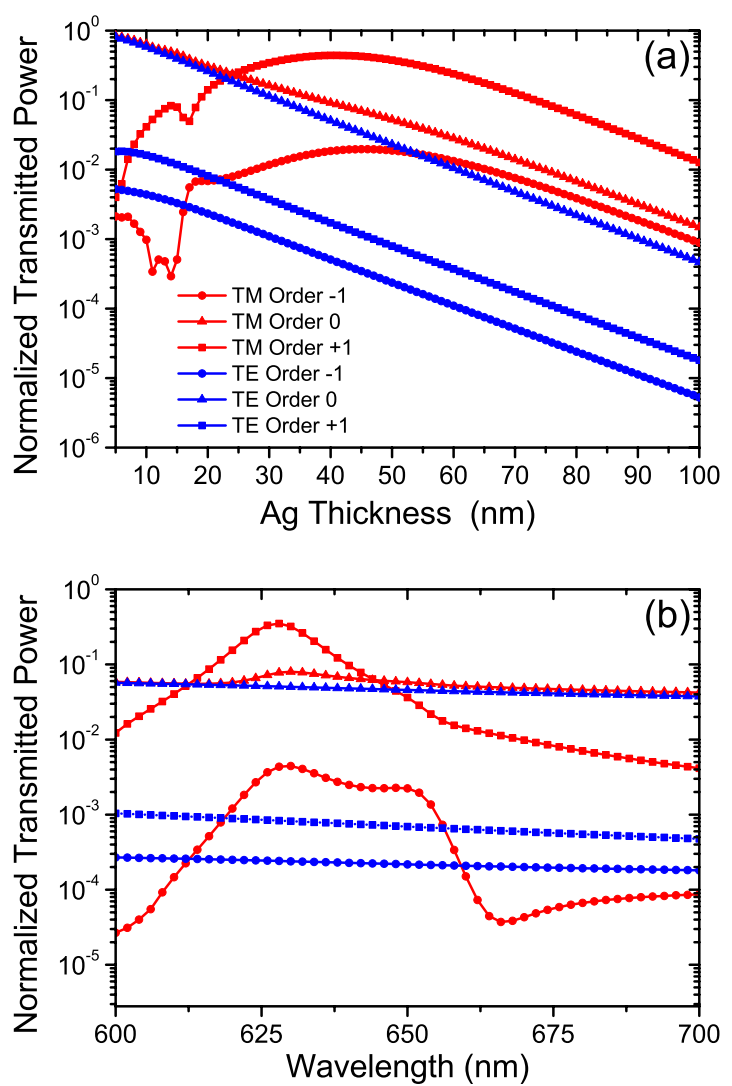

FIG. 2. (Color online) (a) Transmitted fraction of light on resonance as a function of metal thickness for $\theta_{i}=10^{\circ}$ and $\lambda=630 \mathrm{~nm}$. Since TE mode is not resonantly transmitted, the filter also acts as a polarizer, transmitting mainly TM polarization. Optimal Ag thickness is seen to be about $45 \mathrm{~nm}$. (b) Transmitted fraction of light as a function of wavelength for the $-1^{\text {st }}, 0^{\text {th }}$, and $+1^{\text {st }}$ diffraction orders, for incidence angle $\theta_{i}=10^{\circ}$ and $t_{\mathrm{Ag}}=45 \mathrm{~nm}$.

When a collimated beam of light is incident upon a metal grating, resonant coupling to the surface plasmon mode occurs at angles given by ${ }^{14}$

$$
\Theta_{\mathrm{SP}}=\arcsin \left[\operatorname{Re}\left(-\sqrt{\frac{n_{1}^{2} n_{2}^{2}}{n_{1}^{2}+n_{2}^{2}}}\right)+m \frac{\lambda}{n_{1} \Lambda}\right],
$$

where $\Lambda$ is the grating period, $n_{1}$ is the complex index of refraction of the dielectric medium, $n_{2}$ is that of the metal layer and $m$ is an integer. Resonant coupling occurs for only transverse magnetic (TM) polarization (the TM radiation is incident in a plane parallel to the grating wave vector), where the grating vector is parallel to the plane of incidence. ${ }^{15}$ On the other hand, depth and shape of the grating also affects the width of the resonance. ${ }^{16}$

We calculated the transmission intensity through single layer of silver-coated grating with $\Lambda=740 \mathrm{~nm}$ as a function of metal thickness [Fig. 2(a)] and wavelength [Fig. 2(b)] of incoming wave by using rigorously coupled wave analysis (RCWA). ${ }^{17}$ As shown in Fig. 2(a), the transmitted power for $+1^{\text {st }}$ diffraction order reaches its maximum value near $45 \mathrm{~nm}$ silver thickness for TM waves. On the other hand, the transmitted intensity for all the other diffracted orders for transverse electric (TE) and TM waves is an order of magnitude smaller. Figure 2(b) shows wavelength dependence of transmitted power for different diffraction orders of both TE and TM modes. The maximum value of the $+1^{\text {st }}$ diffraction order for TM mode for incidence angle $\theta_{i}=10^{\circ}$ occurs at wavelength $\lambda=628 \mathrm{~nm}$. The filter acts also as a polarizer, with a $\mathrm{TM} / \mathrm{TE}$ polarization ratio of about 100 .

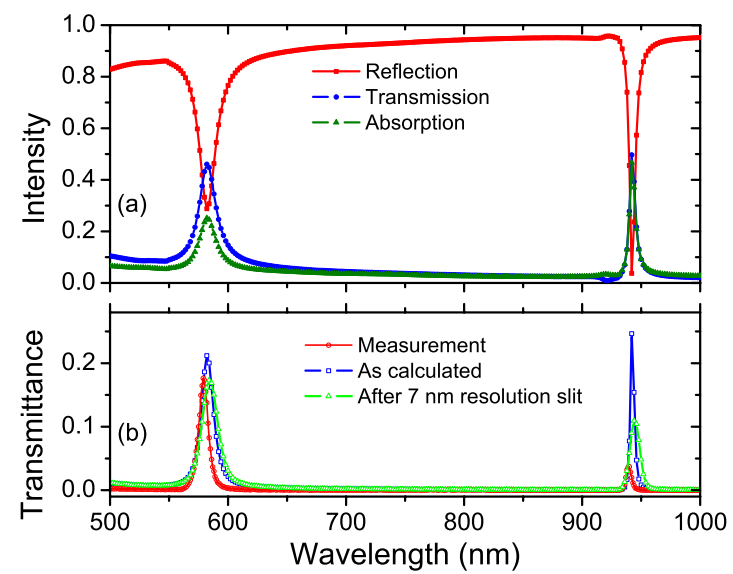

FIG. 3. (Color online) (a) Calculated reflection, transmission and absorption spectra as a function of wavelength for TM waves. Calculation parameters are grating period $\Lambda=740 \mathrm{~nm}$, silver thickness $t_{\mathrm{Ag}}=45 \mathrm{~nm}$ and incidence angle $\theta_{i}=15^{\circ}$. (b) Comparison of measured and calculated transmittance as a function of wavelength. Finite resolution of the spectrometer partially explains the discrepancy between calculations and measurement around $950 \mathrm{~nm}$.

In order to implement the structure, we use gratings present on optical storage disks. Previously, compact disks (CDs) and digital versatile disks (DVDs) have been used in experiments involving grating coupled plasmons. ${ }^{18,19}$ However, to observe sharp resonances, the depth and shape of corrugations must lie within a restricted range. Most commercially available optical disks have corrugated imprinted surfaces protected by external coatings. By exposing the surfaces, simple chemical procedures can be used to tune the surface profile to optimal geometries for sharp and deep plasmon resonance peaks. ${ }^{16}$

We exposed the grating surface of a DVD, by mechanically separating the protective layer. The dye layer can be rinsed using isopropanol or dilute nitric acid, without damaging the grating structure. The grating depth can be adjusted to around $35 \mathrm{~nm}$ by chemically etching the polycarbonate disk in a 1:4 acetone/isopropanol solution for about $45 \mathrm{~s}$. Atomic force microscope (PSIA XE100) was used to characterize the resulting topographies, showing nearly sinusoidal gratings with depths of $35 \mathrm{~nm}$. Two such surfaces are fixed back to back using a thin UV curable epoxy layer. Then the surfaces are metallized by vacuum evaporation of a 45 $\mathrm{nm}$ thick silver layer. A similar procedure was repeated using a $\mathrm{CD}$, where the grating depth was tuned to $60 \mathrm{~nm}$. The transmission through the structures were measured using spectrometric ellipsometers (J. A. Woollam VASE) in the visible and in the near infrared.

The total transmission through the coupled plasmonic grating structure as a function of wavelength for $15^{\circ}$ angle of incidence is calculated by using RCWA and is shown in Fig. 3(a), where $\Lambda=740 \mathrm{~nm}$. The measured transmittance through the filter for $\theta_{i}=15^{\circ}$ shows peak at wavelengths $\lambda$ $=580$ and $940 \mathrm{~nm}$. As shown in Fig. 3(b), the simulations explain the measured spectra quantitatively if a finite spectrometer resolution is assumed.

We measured the transmittance through the proposed plasmonic filter for various incidence angles ranging from $\theta_{i}=0^{\circ}$ to $20^{\circ}$ (Fig. 4). It can be seen that, a peak transmission of up to 0.17 can be achieved over a broad wavelength range, with full width at half maximum of 10 to $5 \mathrm{~nm}$. The positions 


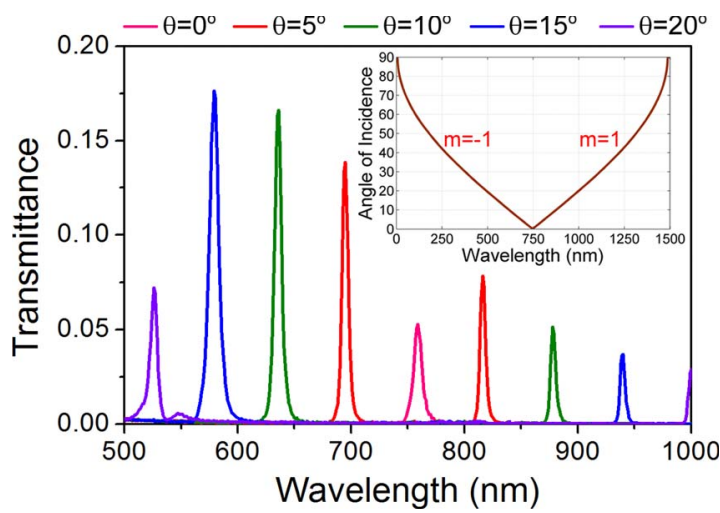

FIG. 4. (Color online) Transmitted intensity of a device having a grating period of $\Lambda=740 \mathrm{~nm}$ and $t=1 \mathrm{~mm}$ at various angles of incidence, as measured with a spectroscopic ellipsometer. No significant transmission for TE waves is observed. The quality factors of transmission peaks are about 100. The inset shows the theoretical results obtained from Eq. (1).

of the peaks agree well with the predictions of RCWA simulations. For instance, the results shown at Fig. 2 predicts the transmission peak to occur at $\lambda=628 \mathrm{~nm}$ for $45 \mathrm{~nm}$ thick $\mathrm{Ag}$ coated grating and $10^{\circ}$ of incidence angle, at the experiment we obtain the transmission peak at $\lambda=635 \mathrm{~nm}$ for the same structure. As shown in the inset in Fig. 2, Eq. (1) predicts the positions of transmission peaks, which agree well with measured results, for a given incidence angle. Two peaks symmetric about $\lambda=750 \mathrm{~nm}$ corresponding to $m=1$ and $m=-1$ are observed ( $n$ and $k$ values are taken from Ref. 20).

For the device fabricated using CDs with grating periods of $\Lambda=1600 \mathrm{~nm}$, PET phenomena can be observed in the near infrared portion of the spectrum at angles of incidence close to normal, as shown in Fig. 5. Due to finite transmission of the CD material (characterized by Fourier transform infrared spectrometer, data not shown), and relatively large thickness of the double CD substrate $(3 \mathrm{~mm})$, total transmission at the resonant wavelength is reduced to few percent. However, well defined resonances with widths of about 50 $\mathrm{nm}$ can be observed. This corresponds to a peak wavelength to width ratio of about 40 , which compares favorably with previously reported PETs at similar wavelengths. ${ }^{21,22}$

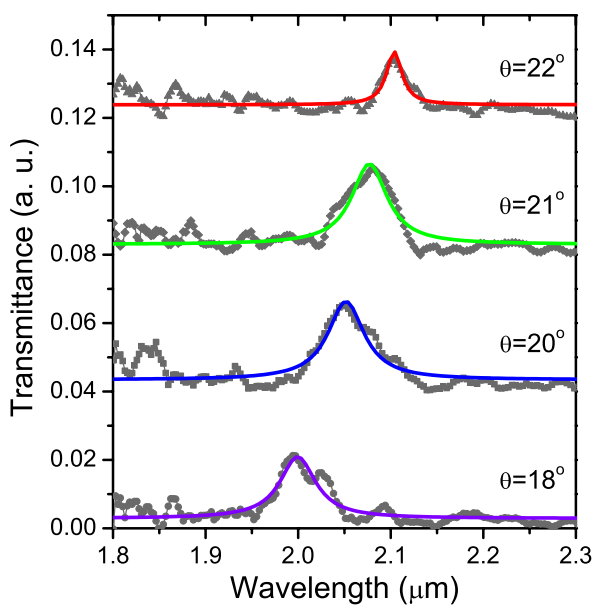

FIG. 5. (Color online) Measured transmitted intensity of a device having a grating period of $\Lambda=1600 \mathrm{~nm}$ at various angles of incidence.
In summary, we proposed and demonstrated an optical filter that uses PET through metallized gratings. The devices are realized using optical disks as starting substrates. Sharp transmission peaks with peak wavelengths dependent on the angle of incidence make the devices suitable for applications in compact spectrometers. By fabricating the device using an elastomeric substrate, peak wavelength can be tuned by applying strain. ${ }^{23,24}$ Theoretical calculations and measurements predict that such devices can be operated at infrared wavelengths by proper scaling. When used to filter collimated thermal emission, devices can be used to fabricate tunable, polarized infrared light sources, or disposable infrared spectrometers. It would be interesting to investigate an array of coupled plasmonic structures by varying the distance between plasmonic gratings. ${ }^{11,12,25}$

This work is supported by TUBITAK under the Grant No. 106T348, 106G090, and 107T547. M.B. acknowledges support from the Turkish Academy of Sciences Distinguished Young Scientist Award (TUBA GEBIP). This work was performed at the UNAM-Institute of Materials Science and Nanotechnology supported by the State Planning Organization of Turkey through the National Nanotechnology Research Center Project.

${ }^{1}$ S. A. Maier and H. A. Atwater, J. Appl. Phys. 98, 011101 (2005).

${ }^{2}$ E. Cubukcu, N. Yu, E. J. Smythe, L. Diehl, K. B. Crozier, and F. Capasso, IEEE J. Sel. Top. Quantum Electron. 14, 1448 (2008).

${ }^{3}$ J. Homola, Anal. Bioanal. Chem. 377, 528 (2003).

${ }^{4}$ T. W. Ebbesen, H. J. Lezec, H. F. Ghaemi, T. Thio, and P. A. Wolff, Nature (London) 391, 667 (1998).

${ }^{5}$ H. F. Ghaemi, T. Thio, D. E. Grupp, T. W. Ebbesen, and H. J. Lezec, Phys. Rev. B 58, 6779 (1998).

${ }^{6}$ A. Giannattasio, I. R. Hooper, and W. L. Barnes, Opt. Express 12, 5881 (2004).

${ }^{7}$ I. Avrutsky, Y. Zhao, and V. Kochergin, Opt. Lett. 25, 595 (2000).

${ }^{8}$ N. Bonod, S. Enoch, L. Li, E. Popov, and M. Nevière, Opt. Express 11, 482 (2003).

${ }^{9}$ Y.-H. Ye and J.-Y. Zhang, Opt. Lett. 30, 1521 (2005).

${ }^{10}$ H. E. Went, A. P. Hibbins, J. R. Sambles, C. R. Lawrence, and A. P. Crick, Appl. Phys. Lett. 77, 2789 (2000).

${ }^{11}$ F. J. Garcia de Abajo, G. Gómez-Santos, L. A. Blanco, A. G. Borisov, and S. V. Shabanov, Phys. Rev. Lett. 95, 067403 (2005).

${ }^{12}$ N. Stefanou, A. Modinos, and V. Yannopapas, Solid State Commun. 118, 69 (2001).

${ }^{13}$ M. Bayindir, E. Cubukcu, I. Bulu, and E. Ozbay, Europhys. Lett. 56, 41 (2001).

${ }^{14}$ M. Kahl and E. Voges, Phys. Rev. B 61, 14078 (2000).

${ }^{15}$ H. Raether, Surface Plasmons on Smooth and Rough Surfaces and on Gratings (Springer, Berlin, 1988).

${ }^{16}$ B. Kaplan, H. Guner, O. Senlik, K. Gurel, M. Bayindir, and A. Dana (unpublished).

${ }^{17}$ International Intellectual Group, Inc., PCGrate. http://www.pcgrate.com.

${ }^{18}$ B. K. Singh and A. C. Hillier, Anal. Chem. 78, 2009 (2006).

${ }^{19}$ E. Fontana, Appl. Opt. 43, 79 (2004).

${ }^{20}$ E. D. Palik, Handbook of Optical Constants of Solids (Academic, New York, 1985), pp. 350-357.

${ }^{21}$ K. Ikeda, H. T. Miyazaki, T. Kasaya, K. Yamamoto, Y. Inoue, K. Fujimura, T. Kanakugi, M. Okada, K. Hatade, and S. Kitagawa, Appl. Phys. Lett. 92, 021117 (2008).

${ }^{22}$ H. T. Miyazaki, K. Ikeda, T. Kasaya, K. Yamamoto, Y. Inoue, K. Fujimura, T. Kanakugi, M. Okada, K. Hatade, and S. Kitagawa, Appl. Phys. Lett. 92, 141114 (2008).

${ }^{23}$ A. Kocabas, F. Ay, A. Dâna, and A. Aydinli, J. Opt. A, Pure Appl. Opt. 8, 85 (2006).

${ }^{24}$ A. Kocabas, A. Dana, and A. Aydinli, Appl. Phys. Lett. 89, 041123 (2006).

${ }^{25}$ M. Bayindir, B. Temelkuran, and E. Ozbay, Phys. Rev. Lett. 84, 2140 (2000). 